\title{
Underwater Superoleophobic Surface Based on Silica Hierarchical Cylinder Arrays with a Low Aspect Ratio
}

\author{
Wendong Liu,* Siyuan Xiang, Xueyao Liu, and Bai Yang*
}

Cite This: ACS Nano 2020, 14, 9166-9175

Read Online

ACCESS |

Llll Metrics \& More

Article Recommendations

Supporting Information

ABSTRACT: A superantiwetting surface based on low-aspectratio hierarchical cylinder arrays (HCAs) was successfully obtained on a silica substrate by colloidal lithography with photolithography. Colloidal lithography is a technique involving transfer of a pattern to a substrate by etching or exposure to a radiation source through a mask composed of a packed colloidal crystal, while photolithography is utilized by which a pattern is transferred photographically to a photoresist-coated substrate, and the substrate is subsequently etched. The surface provides an alternative approach to apply aligned micro-nano

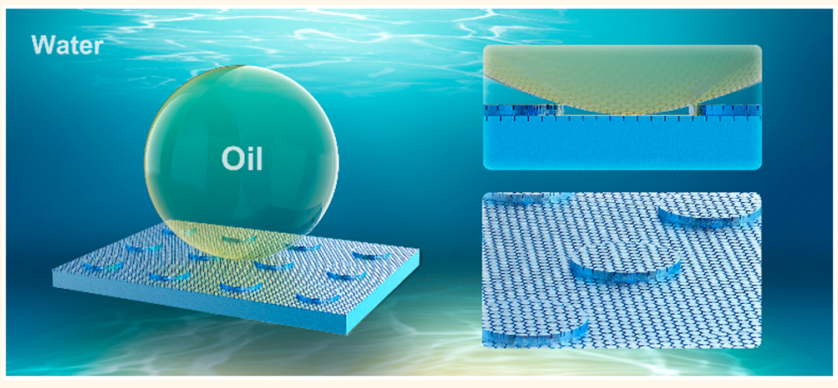
integrated structures with a relatively low aspect ratio in superantiwetting. The obtained HCAs successfully integrated micro- and nanoscale structures into one system, and the physical structure of the HCAs can be tuned by modulating the fabrication approach. Using a postmodification process, the underwater-oil wetting behavior of cylinder-array based surfaces can be easily modulated from the superoleophobic state (an oil contact angle (OCA) of $161^{\circ}$ ) to oleophilic state (an OCA of $19^{\circ}$ ). Moreover, the underwater-oil wettability can be reversibly transformed from the superoleophobic state (an OCA of approximately $153^{\circ}$ ) into the oleophilic state (an OCA of approximately $31^{\circ}$ ) by grafting stimuli-responsive polymer (PNIPAAm) brushes onto this specific hierarchical structure. Due to the temperature-responsive property, modifying the surface with PNIPAAm provides a possibility to control the oil wettability (repellent or sticky) by temperature, which will benefit the use of HCAs in oil-water separation and other application fields.

KEYWORDS: superantiwetting, hierarchical, low aspect ratio, cylinder arrays, colloidal lithography, superoleophobic

$\mathrm{M}$ icro- and nanostructure-dependent superantiwetting has attracted great interest in recent decades due to its specific phenomena in nature. ${ }^{1-5}$ Various micro/ nanoscaled structures have been achieved to mimic these feature-wetting properties since the wettability of the material surface has significant effects on their physicochemical properties (e.g., adhesion, friction, and catalysis). ${ }^{6-8}$ With the deepening understanding of microfabrication techniques and wetting behavior, researchers have turned their attention to the preparation of superantiwetting surfaces, on which the contact angle of droplets is above $150^{\circ}$, to easily realize the rolling and transporting of liquids. These superantiwetting surfaces have great potential for applications in self-cleaning, drag-reduction, ice repellency, antibiofouling, etc. ${ }^{9-12}$ Thus, a series of superhydrophobic surfaces, ${ }^{13,14}$ superoleophobic surfaces, ${ }^{15-17}$ and superamphiphobic surfaces ${ }^{18-21}$ have been fabricated.

Most of the superantiwetting properties of above-mentioned surfaces are based on regulating the liquid wetting behavior by preparing random or ordered micro/nanostructures on the surfaces. ${ }^{15,16,21}$ In contrast, other superantiwetting properties depend on the chemical properties of the materials. ${ }^{17-19}$ Random structure-mediated superantiwetting surfaces can be easily obtained through deposition, template duplication, or surface techniques. However, such a random structure is not a good choice to study wetting behavior due to the inhomogeneous properties of the structure and surfaces. Repeating and regulating a random structure are also very difficult for researchers. ${ }^{22-24}$ On the contrary, ordered micro/ nanostructures fabricated by microfabrication methods (such as photolithography, e-beam lithography, and nanoimprinting

Received: June 4, 2020

Accepted: July 9, 2020

Published: July 9, 2020 
lithography) have become better candidates for evaluating the wetting behavior since their morphologies and feature structure parameters can be easily modulated, while aligned structures can be finely replicated. ${ }^{25-29}$ Thus, various ordered structures, such as nanopost arrays, ${ }^{30-33}$ nanocone arrays, ${ }^{34-37}$ chamfer nanocylinder arrays, ${ }^{38}$ microscale square-pillar arrays, ${ }^{39-41}$ and hierarchical structures, which integrate nanostructures on microstructure arrays, ${ }^{42-44}$ have been fabricated to prepare superantiwetting surfaces.

Nevertheless, most as-prepared ordered structures are prepared with a high aspect ratio. ${ }^{45-48}$ The spaces formed between aligned high-aspect-ratio structures can easily create air pockets or liquid interlayers, which will modulate the wetting behavior into the Cassie state and make it much easier to obtain surfaces with superantiwetting properties. ${ }^{49-51}$ Although structures with a high aspect ratio have found significant applications in the fields such as self-cleaning and antibiofouling, limitations remain. For example, the easy brittle cracking of inorganic materials and the aggregation of organic materials caused by such structures will significantly reduce the wettability and durability of the structured surfaces. ${ }^{52-54}$ Therefore, it is essential to prepare easily fabricated and lowaspect-ratio structure-based superantiwetting surfaces.

In this work, an underwater superoleophobic surface based on low-aspect-ratio silica hierarchical cylinder arrays (HCAs) is fabricated by combining colloidal lithography and photolithography. The feature structure can be modulated by tuning the etching parameters. The wettability of the obtained silica HCAs can transform from an underwater superoleophobic state (an OCA of $161^{\circ}$ ) into an oleophilic state (an OCA of $19^{\circ}$ ) by modifying the structures with specific groups or molecules. The oil wettability transformation from the superoleophobic state (an OCA of approximately $153^{\circ}$ ) into the oleophilic state (an OCA of $31^{\circ}$ ) is realized by modifying the HCAs with a temperature-responsive polymer (PNIPAAm). This work presents an alternative insight for researchers to apply aligned micro-nano integrated structures with relatively low aspect ratios in superantiwetting. Moreover, due to the parallel fabrication, high throughput, and cost efficiency of the combined fabrication methods, it will be beneficial for researchers to apply oleophobic properties based on such hierarchical structures to various substrates and device surfaces for further applications in material, medicine, and biological fields.

\section{RESULTS AND DISCUSSION}

Fabrication of a Silica-HCAs-Based Surface. A silicaHCAs-based surface with a low aspect ratio was fabricated by colloidal lithography with photolithography. The typical fabrication process is shown in Scheme 1. First, photoresist dot arrays were prepared on a silica substrate by a photolithography method. The diameter of the dot was equal to $20 \mu \mathrm{m}$, and the distance between the centers of two adjacent dots' was $40 \mu \mathrm{m}$. Then, the photoresist patterns were employed as masks, and the exposed silica substrate was etched with fluoric plasma to obtain microscale silica cylinder arrays. Second, two-dimensional (2D) hexagonal-close-packed (hcp) polystyrene (PS) microparticles with a diameter of 620 $\mathrm{nm}$ were lifted onto the microscale silica patterns, followed by an oxygen plasma treatment to etch the microparticle arrays into a non-close-packed (ncp) state. Then, nanoscale cylinder arrays were fabricated using the ncp PS arrays as masks to etch the exposed substrate with fluoric plasma to form HCAs, which
Scheme 1. Typical Schematic Procedure to Prepare Silica HCAs with a Low Aspect Ratio
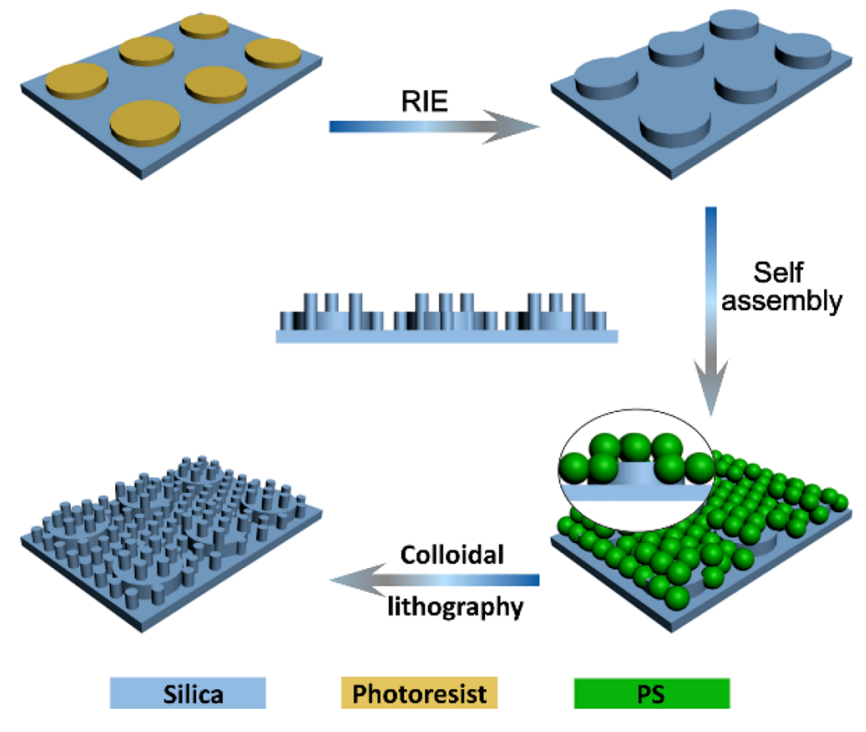

integrated microscale and nanoscale cylinder arrays on the same substrate.

To track the structural evolution, samples of the fabrication stages were characterized using SEM and optical microscopy. The lifted 2D colloidal crystal could reach the entire substrate area, while the finely aligned hcp PS microparticles could reach $100 \mu \mathrm{m}^{2}$ (Figure 1a). PS microparticles occupied over $99 \%$ of the substrate with points and linear defects. This type of largearea orderliness can ensure the quality of the nanoscale silica cylinder arrays. After the PS microparticles were treated with oxygen plasma for $5 \mathrm{~min}$ and further etching of the substrate with fluoric plasma, the hcp microparticle arrays transformed into the ncp state, and the silica substrate beneath became cylinders (Figure 1b). After the maintained PS microparticles were removed, nanoscale silica arrays with a diameter of 423 $\mathrm{nm}$ and a height of $210 \mathrm{~nm}$ were obtained (Figure $1 \mathrm{c}$ and inset), which finely inherited the orders of the hcp masks (Figure S1). Using the ordered microscale and nanoscale cylinder arrays prepared by photolithography and colloidal lithography, silica HCAs over a large area were successfully obtained with the top of each microscale cylinder etched into the nanoscale cylinder arrays (Figure 1d and inset, Figure S2).

To characterize the features of the hierarchical structure, an AFM measurement was conducted. Figure 2 shows a threedimensional (3D) AFM image and the cross-sectional analysis of the HCAs. The height of the microscale cylinder is approximately $560 \mathrm{~nm}$ (Figure 2a). Meanwhile, the top and the adjacent region between microscale cylinders are constructed by orderly aligned nanoscale cylinders (Figure $2 \mathrm{~b}$ ). The aspect ratios of the nanoscale and microscale cylinder are approximately 0.5 and 0.03 , respectively. Thus, low-aspectratio hierarchical cylinders with ordered microscale and nanoscale structures integrated on the same substrate were successfully obtained, and this type of hierarchical structure is a promising material in superwetting substrates, integrated lens, and antireflective surfaces.

Modulation and Optimization of the Structure of Silica HCAs. The hierarchical structure can be modulated through two approaches. The first approach is to modulate the aspect ratio of the nanoscale cylinders by regulating the diameter with a fixed height. Figure 3 presents SEM images of 

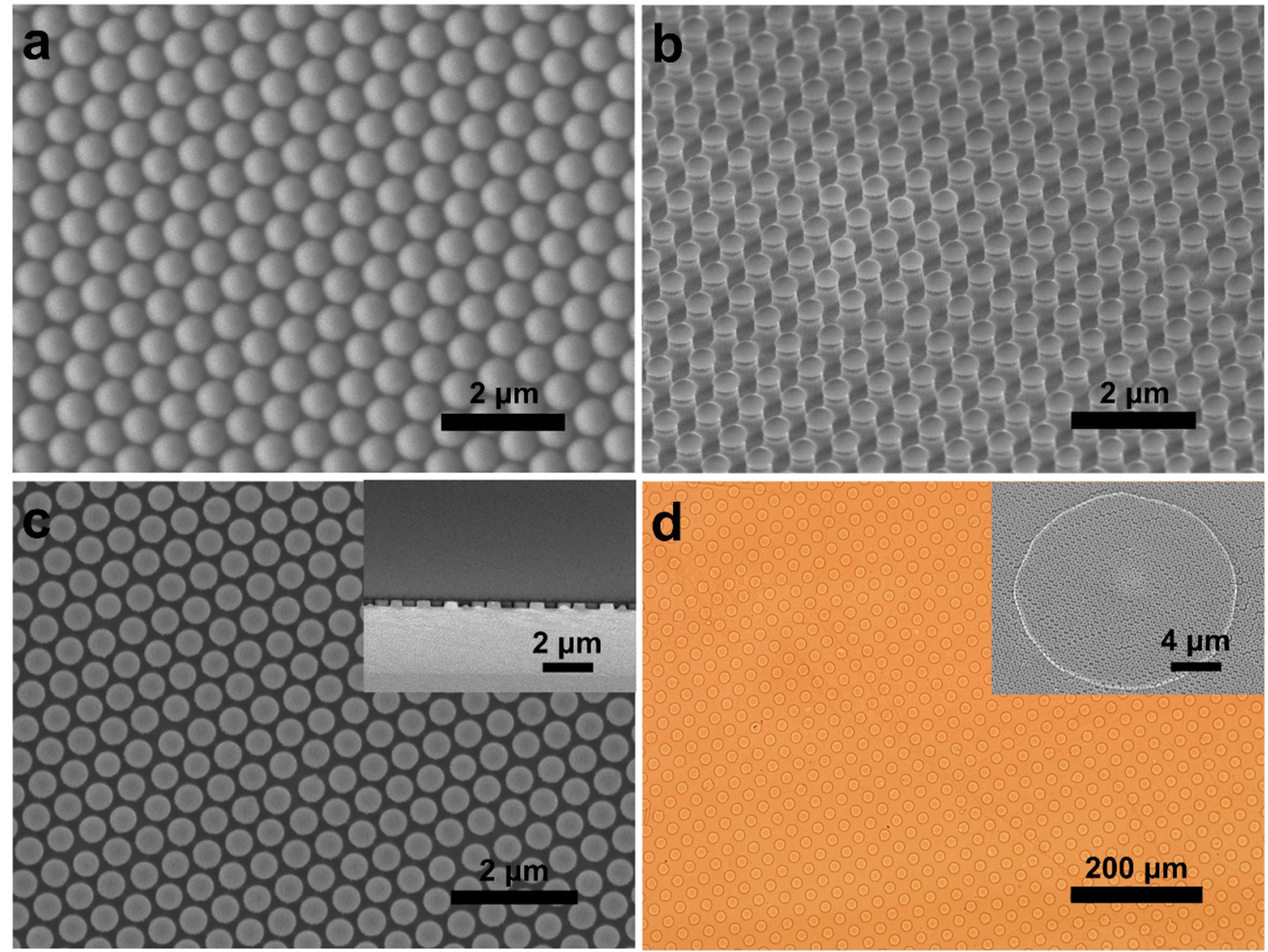

Figure 1. Fabrication of the HCAs-based surface. $(\mathrm{a}-\mathrm{c})$ SEM images of hcp PS microparticles with a diameter of $620 \mathrm{~nm}(\mathrm{a})$, title view of the partially etched silica substrate below the ncp PS microparticles (b), and silica nanocylinder arrays (c); inset is a cross-section view of the nanocylinder. (d) Optical microscopy image of the hierarchical cylinder arrays; the inset shows an SEM image of one microscale cylinder obtained on the substrate.
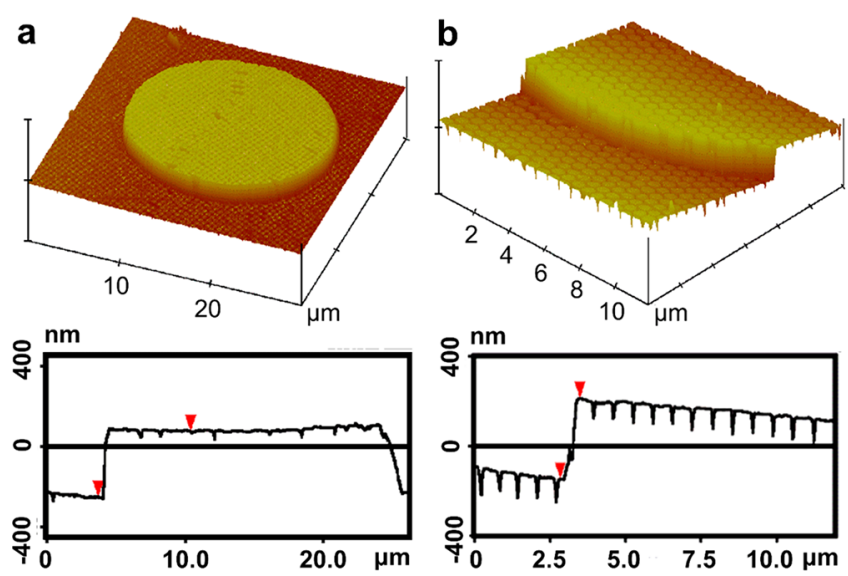

Figure 2. AFM images of the microscale cylinder (a) and nanoscale cylinder arrays constructed hierarchical structure (b). Below are the corresponding cross-sectional analysises; the $z$ scale is $1.2 \mu \mathrm{m}$ and $800 \mathrm{~nm}$.

a series of nanoscale silica cylinder structures. The diameter of the cylinder structures was regulated by adjusting the etching time of the PS microparticles during colloidal lithography processing. After 7, 6, 5, and 4 min of etching followed by 10 min of etching the silica substrate, HCAs with nanoscale cylinder, whose diameters were $240 \mathrm{~nm}$ (a), $350 \mathrm{~nm}$ (b), 423 $\mathrm{nm}$ (c), and $540 \mathrm{~nm}$ (d), were obtained. With increasing etching time, the remained PS microparticles gradually reduced, which created nanoscale cylinder arrays with identical heights but smaller diameters. Finally, the spaces between nanocylinders were progressively increased, which resulted in an adjustable aspect ratio.

The second approach is to fabricate another type of hierarchical cylinder arrays (hCAs) in comparison with the function nanostructures in HCAs. The difference between this arrays and HCAs is that the top of the microscale cylinder is flat and not integrated with the nanoscale cylinders. The necessary fabrication process is shown in Figure 4a. After the microscale silica cylinder arrays were obtained, the photoresist patterns used as masks were retained for further colloidal lithography. The photoresist-protected area will not be further etched; meanwhile, the unprotected space between microscale cylinders will experience a plasma etching process using the assembled PS microparticles as masks, forming nanocylinder arrays. Then, the silica hCAs can be easily obtained after removing the photoresist and remains PS. Figure $4 \mathrm{~b}-\mathrm{c}$ shows 3D AFM images of the hCAs. The microscale cylinder has a flat top surface, whose peripheral was partially etched (Figure S3). This result is ascribed to the inevitable etching procedure, which removes the flash layer of photoresist around the isolated mask and enables the colloidal lithography to proceed on the microscale cylinder. The impact of such defects is negligible due to their small proportion in the entire flat surface. Thus, this type of hCAs will be used to evaluate the role of nanoscale structures in controllable wetting, which is identical to HCAs. Moreover, the diameter of nanocylinder 

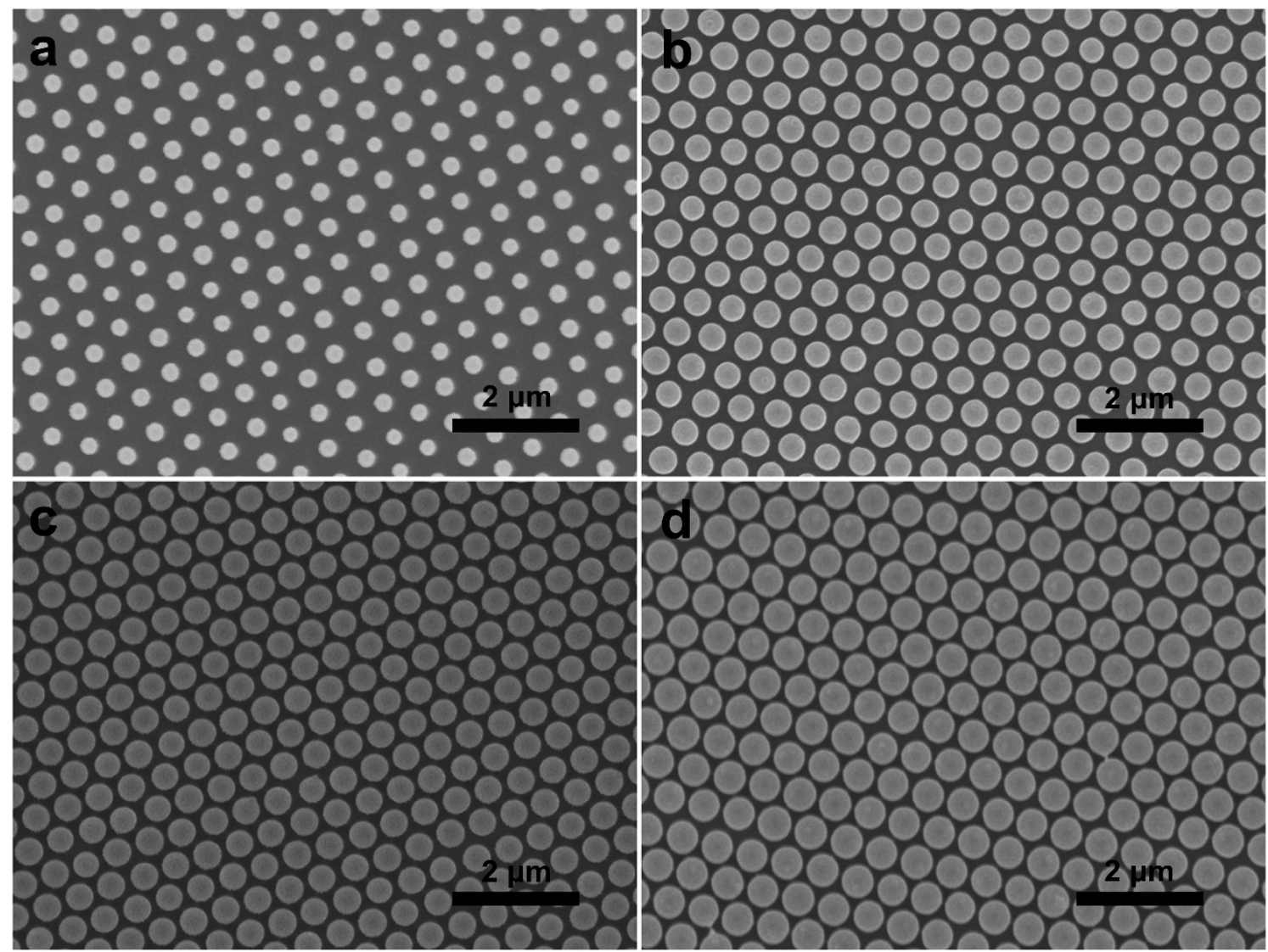

Figure 3. SEM images of nanoscale silica cylinder arrays with diameters of $240 \mathrm{~nm}(\mathrm{a}), 350 \mathrm{~nm}(\mathrm{~b}), 423 \mathrm{~nm}(\mathrm{c})$, and $540 \mathrm{~nm}(\mathrm{~d})$.

arrays between the microscale cylinders can also be modulated by using the aforementioned condition.

After the modulated structures were obtained, wettability was characterized since the micro- or nanostructures significantly affect the properties of these surfaces. Table 1 shows the water contact angles after modifying these hierarchical structures with trichloro $(1 \mathrm{H}, 1 \mathrm{H}, 2 \mathrm{H}, 2 \mathrm{H}$ perfluorooctyl) silane (PFS) to eliminate the effect of the chemical composition. The contact angle data show that the feature structure of the nanocylinder in hCAs directly interacts with the surface wettability. However, with the nanocylinder arrays further integrated to the microscale cylinders, the HCAs exhibited a more hydrophobic wetting behavior than hCAs. The maximal water contact angle was $140^{\circ}$ when the diameter of the nanocylinder was modulated to $423 \mathrm{~nm}$. According to different wettability performances of these regulated hierarchical structures, we can make the following interpretation. Droplets on low-aspect-ratio microscale cylinder arrays exhibit a Wenzel state wetting behavior, where a liquid spreads along the surface since the space between microscale cylinders cannot form intensive air pockets to support droplets in the Cassie state wetting (Figure S4a). For the hCAs, the spaces between microscale cylinders were divided into nanoscale gaps, where the air pockets that formed during liquid wetting were sufficiently strong to support the droplets and caused a Cassie state wetting between microscale cylinders. Meanwhile, a Wenzel state wetting behavior occurred on the surface of microscale cylinders. This wetting behavior changed since the top of the cylinder is flat and unstructured and directly contact with the liquid. Therefore, the hCAs have better hydrophobicity than microscale cylinder arrays since the droplets on this surface presented a Wenzel-Cassie mixed wetting behavior (Figure S4b). For the HCAs, nanocylinder arrays were fully integrated on the entire surface of the microscale cylinder arrays, including the interspaces and the top of microscale cylinders. The nanoscale air pockets formed over the entire surface and completely blocked the Wenzel state wetting, but exhibited the Cassie state wetting behavior (Figure S4c), which implies that the low-aspect-ratio HCAs have the best hydrophobicity. Until, we have successfully improved the wettability of low-aspect-ratio (0.03) microscale cylinder arrays by integrating nanostructures cylinder arrays with an aspect ratio of 0.5 , the surface wettability can be optimized by regulating the feature structure of nanoscale cylinder arrays. (For more information about the wettability of HCAs with nanoscale cylinders (same diameter, but with different aspect ratios), see Supporting Information and Figure S5) For further research, silica HCAs with nanoscale cylinders (diameter of $423 \mathrm{~nm}$ ) was chosen as a model to explore the liquid wetting behavior.

Wetting Behavior of Modified Silica HCAs. Both the surface structure and chemical modifications greatly affect the surface properties, and PFS-functionalized silica HCAs can exhibit hydrophobicity with a water contact angle of $140^{\circ}$. Thus, we further modified the silica HCAs with different small molecules to study whether this type of HCAs could be used for superwetting surfaces.

After being modified with -OH, 3-aminopropyl trimethoxysilane (APTMS), 3-mercaptopropyltrimethoxysilane (MPTMS), octadecyltrichlorosilane (OTCS), or trichloro(octyl)silane (TCOS) through the chemical vapor deposition (CVD) method, the water CA and underwater-oil contact 


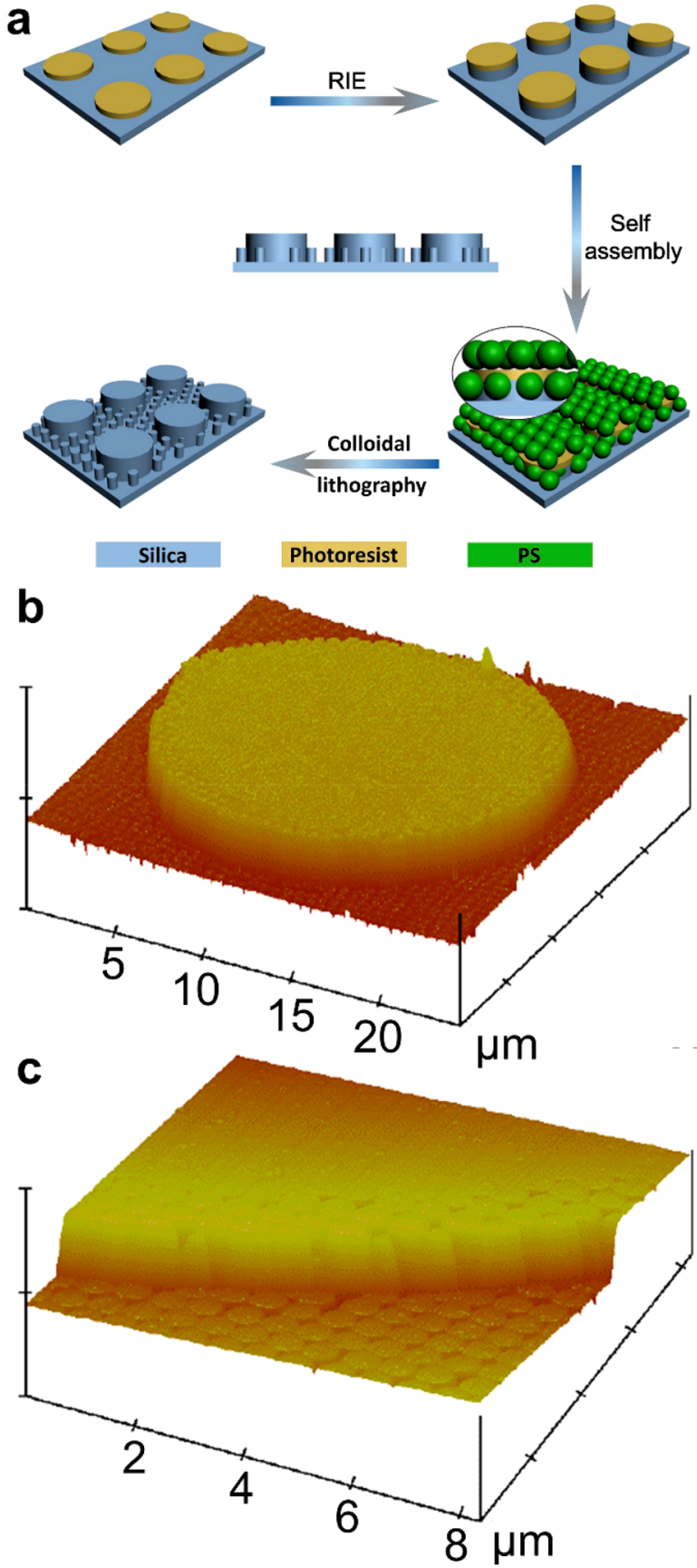

Figure 4. Fabrication of an hCAs-based surface. (a) Typical schematic procedure to prepare silica hCAs with low aspect ratios. During the colloidal lithography process, colloidal particles assembled on the surface will act as a mask to etch the underneath substrate forming into nanocylinder arrays. But for the microscale cylinders, the remained photoresist layer will protect the surface from being etched; thus, no nanocylinders will form on the surface of microscale cylinders. (b,c) 3D AFM images of the microscale cylinder (b) and nanoscale cylinder array constructed hierarchical structure (c); the $z$ scales are 900 and $700 \mathrm{~nm}$.

angle (OCA) were characterized to investigate the wettability of the modified HCAs. Figure 5 shows the water CA and underwater OCA change of the HCAs after they were modified with different molecules, and the corresponding values of the CA in each case are shown in Tables S1 and S2. These results demonstrate that changing the modified molecules with different properties can easily change the wettability of the
Table 1. Water Contact Angle of PFS Modified HCAs and hCAs with Nanoscale Cylinder ${ }^{a}$

\begin{tabular}{cccccc}
\multicolumn{2}{c}{ diameter $(\mathrm{nm})$} & 240 & 350 & 423 & 540 \\
\multirow{2}{*}{ water CA $\left(^{\circ}\right)$} & HCAs & $122 \pm 2$ & $138 \pm 1$ & $\mathbf{1 4 0} \pm \mathbf{2}$ & $135 \pm 2$ \\
& hCAs & $118 \pm 1$ & $134 \pm 2$ & $136 \pm 1$ & $128 \pm 1$
\end{tabular}

${ }^{a}$ Diameters are $240,350,423$, and $540 \mathrm{~nm}$.

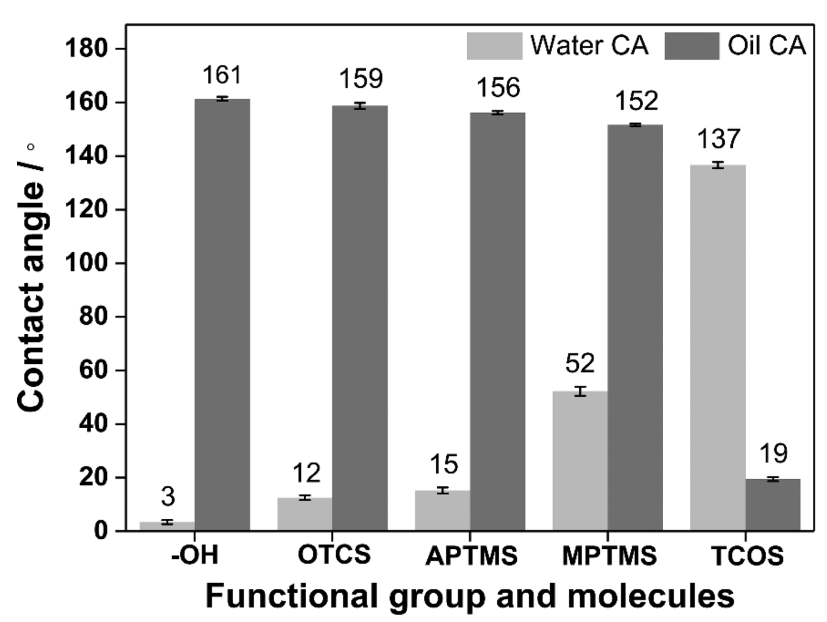

Figure 5. Changes of the water CA and underwater OCA after the silica HCAs were functionalized with specific groups and molecules.

HCAs from a superoleophobic state with an underwater OCA of approximately $161^{\circ}$ into an oleophilic state with the underwater OCA reaching approximately $19^{\circ}$. The water wettability can be transformed from a superhydrophilic state into a hydrophobic state with a controllable water CA value of $3^{\circ}-140^{\circ}$. Thus, using this low-aspect-ratio HCA structure as the substrate, the wetting behavior can be easily regulated. Compared with previous works, ${ }^{45,55,56}$ the aspect ratio of the micro and nanoscale structures was greatly reduced; meanwhile, the wettability maintained. Besides integrating nanocylinder arrays on raised microscale cylinders, it is also suitable to fabricate such kinds of nanostructures on concaved microwells to obtain superoleophobic surfaces (see Supporting Information, Figure S6, and Tables S3 and S4). This type of hierarchical structure, which integrates a microstructure and a nanostructure on the same substrate, provides a foundation to construct low-aspect-ratio structure-based surfaces and can be introduced to various substrates and devices, which have promising practical applications in anti-oil-fouling, selfcleaning, etc. Even though the method presented here can prepare low-aspect-ratio hierarchical structures for super liquid repellent surfaces, the preparation of large-area sample, which can meet the requirement of practical applications, is limited due to the specific fabrication approach. Combining with facile processing methods, such as spin-coating, spray coating, printing, etc. with particle-based building blocks would make it possible to obtain low-aspect-ratio structure-based super liquid repellent surfaces in a fast and cost-efficient manner.

Temperature-Controlled Underwater-Oil Wettability of PNIPAAm Grafted HCAs. Based on the easy functionalization property of the silica HCAs, the structures were modified with poly ( $N$-isopropylacrylamide) (PNIPAAm) by the surface-initiated atom-transfer-radical polymerization (SIATRP) method to prepare a surface with switchable wettability. ${ }^{33}$ For PNIPAAm grafting purposes, the HCAs 
were first treated with oxygen plasma to make them hydrophilic. Then, we covalently bonded the ATRP initiator onto the surface by a two-step method, as demonstrated in the Experimental Methods section. Next, PNIPAAm-functionalized silica HCAs (PNIPAAm-g-HCAs) with different thicknesses were achieved by tuning the polymerization time from 5 to $60 \mathrm{~min}$, and the corresponding film thicknesses were 5, 15, 30, 45, 60, and $90 \mathrm{~nm}$. Figure 6a shows the XPS spectra of the PNIPAAm brush grafted silica HCAs. Peaks of carbon and nitrogen in the spectra are derived from the polymer chain of PNIPAAm, and the corresponding proportion is approximately 5, which proves that the polymer brush film was successfully modified onto the silica cylinder arrays.

Then, an underwater OCA characterization was conducted to study the wettability of the PINPAAm- $g$-HCAs. The relationship between the OCA and the thickness of the PNIPAAm film is shown in Figure 6b. After a relatively thin film of PNIPAAm with a thickness below $30 \mathrm{~nm}$ is coated, the OCA decreases to below $150^{\circ}$. When the thickness increases to 30-50 nm, the PNIPAAm-g-HCAs reach the superoleophobic state (OCA value above $150^{\circ}$ ), and the OCA can reach the highest value near $153^{\circ}$ when the thickness is $45 \mathrm{~nm}$. By further increasing the thickness to $90 \mathrm{~nm}$, we decreased the OCA to below $150^{\circ}$ again. Hence, there is an optimal grafting thickness $(45 \mathrm{~nm})$ of PNIPAAm for these low-aspect-ratio HCAs to maintain the superoleophobicity and further study their wetting transformation behavior under external stimuli. A variotherm underwater OCA measurement was conducted to investigate the transition of oil wettability with temperature variation. Figure $6 \mathrm{c}$ shows that the underwater OCA of PNIPAAM-g-HCAs decreases from $153^{\circ}$ to $31^{\circ}$ when the temperature increases from 20 to $75{ }^{\circ} \mathrm{C}$. The oil wetting behavior can be recovered to the original state by decreasing the temperature, which demonstrates that PNIPAAm-g-HCAs have a reversible transition between superoleophobicity and oleophilicity.

Using the specific stimuli-responsive property of PNIPAAm, the modified HCAs demonstrated a reversible transition of the OCA at different temperatures $\left(20{ }^{\circ} \mathrm{C}\right.$, which is below the lower critical solution temperature (LCST) of PNIPAAm, and $75{ }^{\circ} \mathrm{C}$, which is above the LCST) (Figure 7a), indicating the excellent reproducibility and stability of the wettability transition. Figure $7 \mathrm{~b}$ shows an optical photograph of an oil droplet deposited on the PNIPAAm- $g$-HCAs at 20 and $75^{\circ} \mathrm{C}$. The wettability switching behavior is attributed to the phase transformation of the PNIPAAm brushes with the temperature reciprocating from below to above the LCST, as reported. ${ }^{57,58}$ When the temperature is below the LCST, the PNIPAAm film exhibits a hydrated and swollen state caused by the intermolecular hydrogen bonds with water. The polymer brush film exhibits hydrophilicity and maintains the wetting behavior following the Cassie state. ${ }^{51}$ When the temperature exceeded the LCST, intramolecular hydrogen bonds drive the PNIPAAm film to a shrinking state. At this state, the hydrophobic groups are exposed to the outside, and the polymer brush film presents hydrophobicity, which causes the wetting behavior to transition from the Cassie state into the Wenzel state $^{59}$ and reduces the OCA value to reach approximately $31^{\circ}$. Based on this transition model, the OCA of PNIPAAm- $g$-HCAs demonstrated a reversible transition with changing temperature. These results prove that after PNIPAAm grafting, the oil wettability of the hierarchical structure can freely transform between the superoleophobic
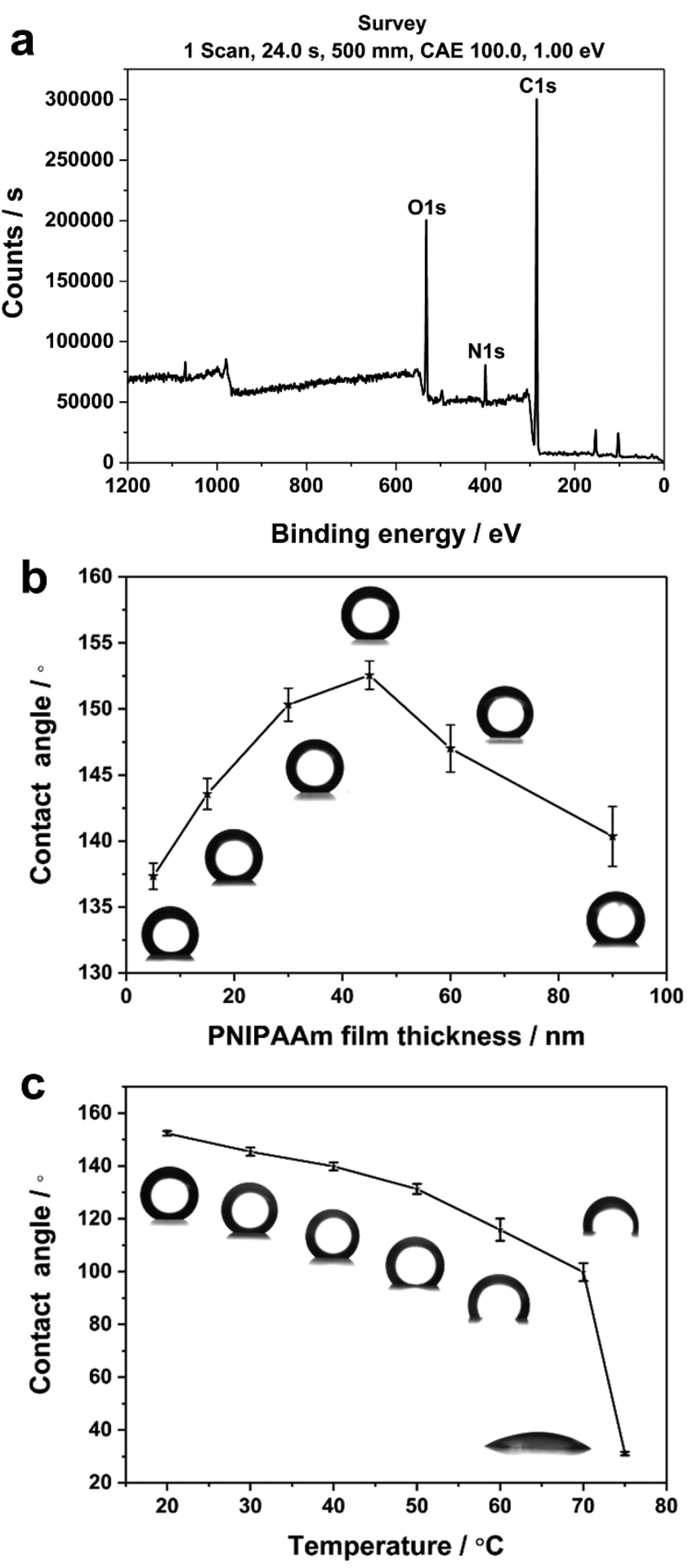

Figure 6. Temperature-responsive PNIPAAm modified HCAs. (a) High-resolution XPS spectra of PNIPAAm coated silica HCAs. (b) Relationship between underwater OCA and the thickness of the PNIPAAm film grafted on silica HCAs. (c) Underwater OCA of the PNIPAAm (film thickness of $45 \mathrm{~nm}$ ) coated silica HCAs as a function of the water temperature.

state (OCA of approximately $153^{\circ}$ ) and the oleophilic state (OCA of approximately $31^{\circ}$ ). Hence, the modified low-aspectratio silica HCAs endowed the micro-nano integrated structures with significant potential for application in the separation of water and oil, filtration, microreactors, lab-onchip devices, etc. 

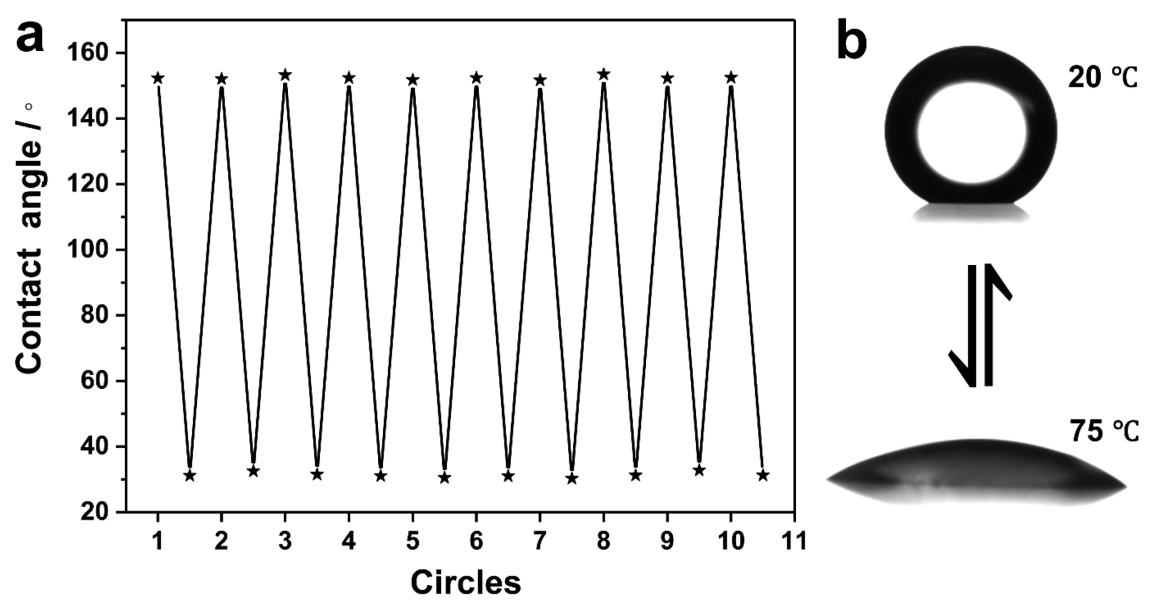

Figure 7. Temperature-controlled underwater-oil wettability transition. (a) Reversible underwater OCA transition of PNIPAAm coated silica HCAs from 20 to $75{ }^{\circ} \mathrm{C}$. (b) Underwater OCA obtained at 20 and $75^{\circ} \mathrm{C}$.

\section{CONCLUSION}

In summary, an underwater superoleophobic surface based on silica hierarchical cylinder arrays with a low aspect ratio is fabricated by the combination of colloidal lithography and photolithography. This surface provides an alternative approach to apply aligned micro-nano integrated structures with a relatively low aspect ratio in superantiwetting. After the structure was coated with an $-\mathrm{OH}$ group or other molecules with specific amphiphilicity, the low-aspect-ratio HCAs exhibited controllable wettability. The wetting behavior of water could transform from a superhydrophilic state (a CA of $3^{\circ}$ ) into a hydrophobic state (a CA of $140^{\circ}$ ). In comparison, the oil wetting behavior could transform from a superoleophobic state (an OCA of $161^{\circ}$ ) into an oleophilic state (an OCA of $19^{\circ}$ ). When the HCAs were functionalized with PNIPAAm, the obtained PNIPAAm- $g$-HCAs exhibited superoleophobicity, and the oil wettability could realize a temperature-controlled reversible transition between the superoleophobic state (an OCA of approximately $153^{\circ}$ ) and the oleophilic state (an OCA of approximately $31^{\circ}$ ). The obtained superoleophobic surface based on low aspect ratio hierarchical structures can help us better understand the wetting behavior, meanwhile providing researchers with another possible way to design a super liquid repellent surface in a cost- and timeefficient manner. Moreover, these structures also show great potential in being applied to various substrate and device surfaces for applications in material, medical, and biological fields.

\section{EXPERIMENTAL METHODS}

Materials. Silica wafers $(2.0 \mathrm{~cm} \times 2.0 \mathrm{~cm})$ were treated in a mixture of $\mathrm{H}_{2} \mathrm{SO}_{4}$ and $30 \% \mathrm{H}_{2} \mathrm{O}_{2}$ (volumetric ratio = 7:3) for $30 \mathrm{~min}$, rinsed with deionized water, and dried under nitrogen gas $\left(\mathrm{N}_{2}\right)$ flow. The $N$-Isopropylacrylamide (NIPAAm) monomer was purchased from J\&K Chemical, $N, N, N^{\prime}, N^{\prime \prime}, N^{\prime \prime}$-pentamethyldiethylenetriamine (PMDETA) was purchased from TCI. Trichloro(octyl)silane (TCOS), octadecyltrichlorosilane (OTCS), 3-aminopropyl trimethoxysilane (APTMS), 3-mercaptopropyl trimethoxysilane (MPTMS), trichloro(1H,1H,2H,2H-perfluorooctyl) silane (PFS), 2bromoisobutyryl bromide, and copper(I) chloride $(\mathrm{CuCl})$ were provided by Aldrich. The polystyrene microparticles with a diameter of $620 \mathrm{~nm}$ were provided by Wuhan Sphere Scientific Co., Ltd. and were cleaned with water and $50 \%$ ethanol several times before use. Dichloromethane, absolute ethanol, triethylamine, sodium dodecyl sulfate (SDS), methanol, 1,2-dichloroethane, and the four compo- nents of the photoresist were used directly after received. The masks employed for photolithography were machined by the Institute of Microelectronics of Chinese Academy of Sciences. The TJ-1A-Micro Flow syringe pump was purchased from Baoding Longer Precision Pump Co., Ltd. The water in all experiments was deionized and doubly distilled prior to use.

Fabrication of Silica Hierarchical Cylinder Arrays by a Combination of Photolithography, Reactive Ion Etching, and Colloidal Lithography. The fabrication of hierarchical cylinder arrays used two steps. First, microscale cylinder arrays were prepared via photolithography and reactive ion etching (RIE). Second, nanoscale cylinders were fabricated on the microscale patterns to achieve hierarchical structures. The detailed fabrication process was as follows: The cleaned silica substrate was spin coated with a layer of positive photoresist. Then the photoresist layer was patterned by traditional photolithography techniques using a mask with square dot arrays. The diameter of the dot was $20 \mu \mathrm{m}$, and the distance between the centers of two adjacent two dots was $40 \mu \mathrm{m}$. After developing the patterned photoresist following the exposure to UV light, we used the maintained photoresist dot arrays as a mask to conduct RIE. The etching process was proceeded using Plasmalab 80 Plus (Oxford Instrument) at $30 \mathrm{mTorr}$ pressure, $35 \mathrm{SCCM} \mathrm{CHF}_{3}$ and a $15 \mathrm{SCCM}$ Ar mixed gas flow rate, RF power of $250 \mathrm{~W}$, and ICP power of $0 \mathrm{~W}$ for $20 \mathrm{~min}$. The microscale silica cylinder arrays were obtained by rinsing the substrates with acetone and absolute ethanol to remove the remaining photoresist and dried with an $\mathrm{N}_{2}$ stream.

Low-aspect-ratio hierarchical cylinder arrays (HCAs), which integrated nanoscale cylinder arrays on the microscale patterns, were fabricated by colloidal lithography. First, 2D colloidal crystals were self-assembled onto the silica substrate with microscale cylinders using the interfacial modification method as reported. ${ }^{33}$ The diameter of the polystyrene (PS) microparticles in this work was $620 \mathrm{~nm}$. Then, the nanoscale cylinder arrays were prepared via a two-step RIE process: After the $2 \mathrm{D}$ hcp colloidal crystals were achieved, hexagonalclose-packed (hcp) microparticles were etched into a non-closepacked state (ncp) by oxygen RIE. The RIE was operated at 10 mTorr pressure, a $50 \mathrm{SCCM}$ oxygen gas flow rate, RF power of $60 \mathrm{~W}$, and ICP power of $0 \mathrm{~W}$ for 4, 5, 6, and $7 \mathrm{~min}$. Then, the retained ncp PS microparticle arrays acted as the masks, and the exposed silica substrate was further etched with fluoric plasma by operating the RIE

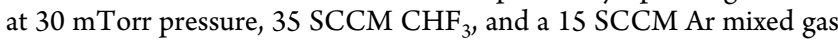
flow rate, and RF power $250 \mathrm{~W}$, ICP power $0 \mathrm{~W}$ for $10 \mathrm{~min}$. Then, low-aspect-ratio silica HCAs were obtained after washing off the remaining PS masks with toluene.

To confirm the effect of nanoscale cylinder arrays on the wetting behavior, another type of hierarchical cylinder arrays (hCAs) was fabricated. The hCAs had a specific structure with the space between microscale cylinders etched into nanoscale cylinders, while the microscale cylinders were not further structured. To obtain this 
type of structure, the photoresist used as a mask to prepare microscale cylinders was not washed off. Then, the $2 \mathrm{D}$ colloidal crystal was directly assembled on the surface. Using the aforementioned method, nanoscale cylinder arrays were fabricated on exposed the substrate between neighboring microscale cylinders. Meanwhile, the microscale cylinder was protected from further etching. After the etching process, low-aspect-ratio silica hCAs were obtained by removing the remained photoresist and PS masks with toluene.

Modifying Silica HCAs and hCAs with Different Groups and Molecules. After the silica HCAs and hCAs were obtained, the structured surfaces could easily be altered and coated with diverse groups and molecules to study the surface wettability. For modification, the silica-based surfaces were first treated with oxygen plasma to hydroxylate the surfaces. Then, PFS, TCOS, OTCS, APTMS, and MPTMS could be grafted onto the surfaces by chemical vapor deposition through covalent bonding. In brief, the silica wafers were placed in a sealed vessel into which a few drops of silane (PFS, TCOS, OTCS, APTMS, and MPTMS) were deposited onto the bottom, maintaining the wafers and drops separation. Then the vessel was heated at $60{ }^{\circ} \mathrm{C}$ for $1 \mathrm{~h}$ to covalently graft silanes onto the substrates. To coat the HCAs with macromolecules, $\operatorname{poly}(\mathrm{N}$ isopropylacrylamide) (PNIPAAm) was chosen as a model material to modify the surface of HCAs via surface-initiated atom-transfer radical polymerization (SI-ATRP) as described. ${ }^{36}$

Characterization. SEM images were taken using a JEOL FESEM $6700 \mathrm{~F}$ electron microscope with a primary electron energy of $3 \mathrm{kV}$. The substrates were sputter-coated with a $5 \mathrm{~nm}$ layer of $\mathrm{Pt}$ prior to taking images. The thickness of the PNIPAAm film was measured by using a Dektak 150 surface profiler (Veeco). Atomic force microscopy (AFM) images were recorded in the tapping mode with a Nanoscope III scanning probe microscope from Digital Instruments. The optical microscopy image was taken by an OLYMPUS BX51. X-ray photoelectron spectroscopy (XPS) was characterized by using an ESCALAB 250 spectrometer with a mono X-ray source $\mathrm{Al} \mathrm{Ka}$ excitation $(1486.6 \mathrm{eV})$. The oil (1,2-dichloroethane) contact angles underwater were investigated using Dataphysics OCA20. A $5 \mu \mathrm{L}$ oil droplet was deposited onto the samples immersed in water, and the static contact angle (CA) was employed as the indicator to evaluate the wettability of the hierarchical structures, as determined by the average value, which was measured at least three times at different positions on each sample. The water CA was measured with a $5 \mu \mathrm{L}$ of water droplet deposited onto the samples in the air.

\section{ASSOCIATED CONTENT}

\section{(s) Supporting Information}

The Supporting Information is available free of charge at https://pubs.acs.org/doi/10.1021/acsnano.0c04670.

Supplementary water contact angle (CA) and underwater-oil contact angle (OCA) of modified HCAs and hierarchical well arrays; SEM images of the silica HCAs and hCAs; SEM and AFM images of hierarchical well arrays; schematic wetting of the droplet of water deposited on the microscale cylinder arrays, hCAs, and HCAs (PDF)

\section{AUTHOR INFORMATION}

\section{Corresponding Authors}

Wendong Liu - State Key Laboratory of Supramolecular Structure and Materials, College of Chemistry, Jilin University, Changchun 130012, People's Republic of China; Max Planck Institute for Polymer Research, D-55128 Mainz, Germany; ○ orcid.org/0000-0001-7297-8591; Email: liuwendong@ mpip-mainz.mpg.de

Bai Yang - State Key Laboratory of Supramolecular Structure and Materials, College of Chemistry, Jilin University,
Changchun 130012, People's Republic of China; 자이.org/ 0000-0002-3873-075X; Email: byangchem@jlu.edu.cn

\section{Authors}

Siyuan Xiang - State Key Laboratory of Supramolecular Structure and Materials, College of Chemistry, Jilin University, Changchun 130012, People's Republic of China; Max Planck Institute for Polymer Research, D-55128 Mainz, Germany

Xueyao Liu - State Key Laboratory of Supramolecular Structure and Materials, College of Chemistry, Jilin University, Changchun 130012, People's Republic of China

Complete contact information is available at:

https://pubs.acs.org/10.1021/acsnano.0c04670

\section{Author Contributions}

W.L. and B.Y. conceived and designed the experiments. W.L., S.X., and X.L. performed the experiments and characterizations. W.L., S.X., X.L., and B.Y. cowrote the paper. All authors discussed the results and commented on the manuscript.

Notes

The authors declare no competing financial interest.

\section{ACKNOWLEDGMENTS}

This work was financially supported by the National Basic Research Program of China (973 program) under grant no. 2012CB933800, and the National Science Foundation of China (NSFC) under grant no. 91123031.

\section{REFERENCES}

(1) Liu, M.; Wang, S.; Jiang, L. Nature-Inspired Superwettability Systems. Nat. Rev. Mater. 2017, 2, 17036.

(2) E, J.; Jin, Y.; Deng, Y.; Zuo, W.; Zhao, X.; Han, D.; Peng, Q.; Zhang, Z. Wetting Models and Working Mechanisms of Typical Surfaces Existing in Nature and Their Application on Superhydrophobic Surfaces: A Review. Adv. Mater. Interfaces 2018, 5, 1701052.

(3) Zhang, S.; Huang, J.; Chen, Z.; Lai, Y. Bioinspired Special Wettability Surfaces: From Fundamental Research to Water Harvesting Applications. Small 2017, 13, 1602992.

(4) Darmanin, T.; Guittard, F. Superhydrophobic and Superoleophobic Properties in Nature. Mater. Today 2015, 18, 273-285.

(5) Liu, K.; Jiang, L. Bio-Inspired Design of Multiscale Structures for Function Integration. Nano Today 2011, 6, 155-175.

(6) Wen, G.; Guo, Z.; Liu, W. Biomimetic Polymeric Superhydrophobic Surfaces and Nanostructures: From Fabrication to Applications. Nanoscale 2017, 9, 3338-3366.

(7) Tian, Y.; Su, B.; Jiang, L. Interfacial Material System Exhibiting Superwettability. Adv. Mater. 2014, 26, 6872-6897.

(8) Liu, M.; Zheng, Y.; Zhai, J.; Jiang, L. Bioinspired SuperAntiwetting Interfaces with Special Liquid-Solid Adhesion. Acc. Chem. Res. 2010, 43, 368-377.

(9) Zheng, K.; Zhang, J.; Dodiuk, H.; Kenig, S.; Barry, C.; Sun, H.; Mead, J. Effect of Superhydrophobic Composite Coatings on Drag Reduction in Laminar Flow. ACS Appl. Polym. Mater. 2020, 2, 16141622.

(10) Khanmohammadi Chenab, K.; Sohrabi, B.; Rahmanzadeh, A. Superhydrophobicity: Advanced Biological and Biomedical Applications. Biomater. Sci. 2019, 7, 3110-3137.

(11) Gogolides, E.; Ellinas, K.; Tserepi, A. Hierarchical Micro and Nano Structured, Hydrophilic, Superhydrophobic and Superoleophobic Surfaces Incorporated in Microfluidics, Microarrays and Lab on Chip Microsystems. Microelectron. Eng. 2015, 132, 135-155.

(12) Zhang, W.; Lin, G.; Li, J.; Xue, H.; Luo, Y.; Gao, X. Fabrication of Biomimetic Polymer Nanocone Films with Condensate Microdrop Self-Removal Function. Adv. Mater. Interfaces 2015, 2, 1500238. 
(13) Geyer, F.; D’Acunzi, M.; Sharifi-Aghili, A.; Saal, A.; Gao, N.; Kaltbeitzel, A.; Sloot, T.-F.; Berger, R.; Butt, H.-J.; Vollmer, D. When and How Self-Cleaning of Superhydrophobic Surfaces Works. Sci. Adv. 2020, 6, No. eaaw9727.

(14) Wu, D.; Wu, S.-Z.; Chen, Q.-D.; Zhang, Y.-L.; Yao, J.; Yao, X.; Niu, L.-G.; Wang, J.-N.; Jiang, L.; Sun, H.-B. Curvature-Driven Reversible In Situ Switching between Pinned and Roll-Down Superhydrophobic States for Water Droplet Transportation. Adv. Mater. 2011, 23, 545-549.

(15) Yong, J.; Chen, F.; Yang, Q.; Huo, J.; Hou, X. Superoleophobic Surfaces. Chem. Soc. Rev. 2017, 46, 4168-4217.

(16) Cai, Y.; Lin, L.; Xue, Z.; Liu, M.; Wang, S.; Jiang, L. FilefishInspired Surface Design for Anisotropic Underwater Oleophobicity. Adv. Funct. Mater. 2014, 24, 809-816.

(17) Yao, X.; Gao, J.; Song, Y.; Jiang, L. Superoleophobic Surfaces with Controllable Oil Adhesion and Their Application in Oil Transportation. Adv. Funct. Mater. 2011, 21, 4270-4276.

(18) Zhou, H.; Wang, H.; Niu, H.; Zhao, Y.; Xu, Z.; Lin, T. A Waterborne Coating System for Preparing Robust, Self-Healing, Superamphiphobic Surfaces. Adv. Funct. Mater. 2017, 27, 1604261.

(19) Chu, Z.; Seeger, S. Superamphiphobic Surfaces. Chem. Soc. Rev. 2014, 43, 2784-2798.

(20) Peng, S.; Yang, X.; Tian, D.; Deng, W. Chemically Stable and Mechanically Durable Superamphiphobic Aluminum Surface with a Micro/Nanoscale Binary Structure. ACS Appl. Mater. Interfaces 2014, 6, 15188-15197.

(21) Deng, X.; Mammen, L.; Butt, H.-J.; Vollmer, D. Candle Soot as a Template for a Transparent Robust Superamphiphobic Coating. Science 2012, 335, 67-70.

(22) Ma, W.; Xu, H.; Takahara, A. Substrate-Independent Underwater Superoleophobic Surfaces Inspired by Fish-Skin and Mussel-Adhesives. Adv. Mater. Interfaces 2014, 1, 1300092.

(23) Bellanger, H.; Darmanin, T.; Taffin de Givenchy, E.; Guittard, F. Robustness Tests on Superoleophobic Pedop Films. Colloids Surf., A 2013, 433, 47-54.

(24) Liu, X.; Zhou, J.; Xue, Z.; Gao, J.; Meng, J.; Wang, S.; Jiang, L. Clam's Shell Inspired High-Energy Inorganic Coatings with Underwater Low Adhesive Superoleophobicity. Adv. Mater. 2012, 24, 3401-3405.

(25) Sun, Y.; Guo, Z. Recent Advances of Bioinspired Functional Materials with Specific Wettability: From Nature and Beyond Nature. Nanoscale Horiz. 2019, 4, 52-76.

(26) Liu, W.; Liu, X.; Ge, P.; Fang, L.; Xiang, S.; Zhao, X.; Shen, H.; Yang, B. Hierarchical-Multiplex DNA Patterns Mediated by Polymer Brush Nanocone Arrays That Possess Potential Application for Specific DNA Sensing. ACS Appl. Mater. Interfaces 2015, 7, 2476024771

(27) Liu, W.; Li, Y.; Wang, T.; Li, D.; Fang, L.; Zhu, S.; Shen, H.; Zhang, J.; Sun, H.; Yang, B. Elliptical Polymer Brush Ring Array Mediated Protein Patterning and Cell Adhesion on Patterned Protein Surfaces. ACS Appl. Mater. Interfaces 2013, 5, 12587-12593.

(28) Zhang, G.; Wang, D. Colloidal Lithography-The Art of Nanochemical Patterning. Chem. - Asian J. 2009, 4, 236-245.

(29) Christman, K. L.; Schopf, E.; Broyer, R. M.; Li, R. C.; Chen, Y.; Maynard, H. D. Positioning Multiple Proteins at the Nanoscale with Electron Beam Cross-Linked Functional Polymers. J. Am. Chem. Soc. 2009, 131, 521-527.

(30) Papadopoulos, P.; Pinchasik, B.-E.; Tress, M.; Vollmer, D.; Kappl, M.; Butt, H.-J. Wetting of Soft Superhydrophobic Micropillar Arrays. Soft Matter 2018, 14, 7429-7434.

(31) Chen, Y.; Meng, J.; Zhu, Z.; Zhang, F.; Wang, L.; Gu, Z.; Jiang, L.; Wang, S. Controlled Growth of Patterned Conducting Polymer Microsuckers on Superhydrophobic Micropillar-Structured Templates. Adv. Funct. Mater. 2018, 28, 1800240.

(32) Zeniou, A.; Ellinas, K.; Olziersky, A.; Gogolides, E. Ultra-High Aspect Ratio Si Nanowires Fabricated with Plasma Etching: Plasma Processing, Mechanical Stability Analysis against Adhesion and Capillary Forces and Oleophobicity. Nanotechnology 2014, 25, No. 035302
(33) Xu, X.; Vereecke, G.; Chen, C.; Pourtois, G.; Armini, S.; Verellen, N.; Tsai, W.-K.; Kim, D.-W.; Lee, E.; Lin, C.-Y.; Van Dorpe, P.; Struyf, H.; Holsteyns, F.; Moshchalkov, V.; Indekeu, J.; De Gendt, S. Capturing Wetting States in Nanopatterned Silicon. ACS Nano 2014, 8, 885-893.

(34) Li, H.; Chen, F.-H.; Biria, S.; Hosein, I. D. Prototyping of Superhydrophobic Surfaces from Structure-Tunable Micropillar Arrays Using Visible Light Photocuring. Adv. Eng. Mater. 2019, 21, 1801150.

(35) Wang, W.; Salazar, J.; Vahabi, H.; Joshi-Imre, A.; Voit, W. E.; Kota, A. K. Metamorphic Superomniphobic Surfaces. Adv. Mater. 2017, 29, 1700295.

(36) Liu, W.; Liu, X.; Fangteng, J.; Wang, S.; Fang, L.; Shen, H.; Xiang, S.; Sun, H.; Yang, B. Bioinspired Polyethylene Terephthalate Nanocone Arrays with Underwater Superoleophobicity and AntiBioadhesion Properties. Nanoscale 2014, 6, 13845-13853.

(37) Toma, M.; Loget, G.; Corn, R. M. Flexible Teflon Nanocone Array Surfaces with Tunable Superhydrophobicity for Self-Cleaning and Aqueous Droplet Patterning. ACS Appl. Mater. Interfaces 2014, 6, 11110-11117.

(38) Liu, W.; Liu, X.; Xiang, S.; Chen, Y.; Fang, L.; Yang, B. Functional Interface Based on Silicon Artificial Chamfer Nanocylinder Arrays (CNCAs) with Underwater Superoleophobicity and Anisotropic Properties. Nano Res. 2016, 9, 3141-3151.

(39) Zhang, R.; Hao, P.; Zhang, X.; Niu, F.; He, F. Tunable Droplet Breakup Dynamics on Micropillared Superhydrophobic Surfaces. Langmuir 2018, 34, 7942-7950.

(40) Su, B.; Wang, S.; Ma, J.; Wu, Y.; Chen, X.; Song, Y.; Jiang, L. Elaborate Positioning of Nanowire Arrays Contributed by Highly Adhesive Superhydrophobic Pillar-Structured Substrates. Adv. Mater. 2012, 24, 559-564.

(41) Liu, M.; Wang, S.; Wei, Z.; Song, Y.; Jiang, L. Bioinspired Design of a Superoleophobic and Low Adhesive Water/Solid Interface. Adv. Mater. 2009, 21, 665-669.

(42) Yang, Y.; Li, X.; Zheng, X.; Chen, Z.; Zhou, Q.; Chen, Y. 3DPrinted Biomimetic Super-Hydrophobic Structure for Microdroplet Manipulation and Oil/Water Separation. Adv. Mater. 2018, 30, 1704912.

(43) Wooh, S.; Koh, J. H.; Lee, S.; Yoon, H.; Char, K. TrilevelStructured Superhydrophobic Pillar Arrays with Tunable Optical Functions. Adv. Funct. Mater. 2014, 24, 5550-5556.

(44) Marquez-Velasco, J.; Vlachopoulou, M.-E.; Tserepi, A.; Gogolides, E. Stable Superhydrophobic Surfaces Induced by DualScale Topography on SU-8. Microelectron. Eng. 2010, 87, 782-785.

(45) Ma, Z.; Jiang, C.; Li, X.; Ye, F.; Yuan, W. Controllable Fabrication of Periodic Arrays of High-Aspect-Ratio Micro-Nano Hierarchical Structures and Their Superhydrophobicity. J. Micromech. Microeng. 2013, 23, No. 095027.

(46) Higgins, S. G.; Becce, M.; Belessiotis-Richards, A.; Seong, H.; Sero, J. E.; Stevens, M. M. High-Aspect-Ratio Nanostructured Surfaces as Biological Metamaterials. Adv. Mater. 2020, 32, 1903862.

(47) Chen, C.-M.; Yang, S. Directed Water Shedding on HighAspect-Ratio Shape Memory Polymer Micropillar Arrays. Adv. Mater. 2014, 26, 1283-1288.

(48) Leber, M.; Shandhi, M. M. H.; Hogan, A.; Solzbacher, F.; Bhandari, R.; Negi, S. Different Methods to Alter Surface Morphology of High Aspect Ratio Structures. Appl. Surf. Sci. 2016, 365, 180-190.

(49) Marchio, S.; Meloni, S.; Giacomello, A.; Casciola, C. M. Wetting and Recovery of Nano-Patterned Surfaces Beyond the Classical Picture. Nanoscale 2019, 11, 21458-21470.

(50) Neckernuss, T.; Wiedemann, S.; Plettl, A.; Ziemann, P. Moving Water Droplets over Nanoscaled (Super) Hydrophobic Wettability Contrasts: Experimental Test of a Simple Model Describing Driving Forces. Adv. Mater. Interfaces 2014, 1, 1300033.

(51) Cassie, A. B. D.; Baxter, S. Wettability of Porous Surfaces. Trans. Faraday Soc. 1944, 40, 546-551.

(52) Roca-Cusachs, P.; Rico, F.; Martínez, E.; Toset, J.; Farré, R.; Navajas, D. Stability of Microfabricated High Aspect Ratio Structures in Poly(Dimethylsiloxane). Langmuir 2005, 21, 5542-5548. 
(53) Chandra, D.; Yang, S. Stability of High-Aspect-Ratio Micropillar Arrays against Adhesive and Capillary Forces. Acc. Chem. Res. 2010, 43, 1080-1091.

(54) Vrancken, N.; Vereecke, G.; Bal, S.; Sergeant, S.; Doumen, G.; Holsteyns, F.; Terryn, H.; de Gendt, S.; Xu, X. M. Pattern Collapse of High-Aspect-Ratio Silicon Nanostructures - a Parametric Study. Solid State Phenom. 2016, 255, 136-140.

(55) Zhou, M.; Xiong, X.; Jiang, B.; Weng, C. Fabrication of High Aspect Ratio Nanopillars and Micro/Nano Combined Structures with Hydrophobic Surface Characteristics by Injection Molding. Appl. Surf. Sci. 2018, 427, 854-860.

(56) Patankar, N. A. Mimicking the Lotus Effect: Influence of Double Roughness Structures and Slender Pillars. Langmuir 2004, 20, 8209-8213.

(57) Liu, H.; Zhang, X.; Wang, S.; Jiang, L. Underwater Thermoresponsive Surface with Switchable Oil-Wettability between Superoleophobicity and Superoleophilicity. Small 2015, 11, 33383342.

(58) Xue, B.; Gao, L.; Hou, Y.; Liu, Z.; Jiang, L. Temperature Controlled Water/Oil Wettability of a Surface Fabricated by a Block Copolymer: Application as a Dual Water/Oil On-Off Switch. Adv. Mater. 2013, 25, 273-277.

(59) Wenzel, R. N. Resistance of Solid Surfaces to Wetting by Water. Ind. Eng. Chem. 1936, 28, 988-994. 